\title{
Nonlinear oscillations of a sessile drop on a hydrophobic surface induced by ac electrowetting
}

\author{
Joohee Lee, ${ }^{1}$ Jun Kwon Park,,${ }^{2,}$ Jiwoo Hong, ${ }^{2}$ Sang Joon Lee, ${ }^{2}$ Kwan Hyoung Kang, ${ }^{2}$ and Hyung Ju Hwang ${ }^{3, \dagger}$ \\ ${ }^{1}$ Department of Mathematics, Chung-Ang University, Seoul 156-756, Republic of Korea \\ ${ }^{2}$ Department of Mechanical Engineering, Pohang University of Science and Technology, Pohang 790-784, Republic of Korea \\ ${ }^{3}$ Department of Mathematics, Pohang University of Science and Technology, Pohang 790-784, Republic of Korea
}

(Received 16 July 2014; published 30 September 2014)

\begin{abstract}
We examine the nature of ac electrowetting (EW)-driven axisymmetric oscillations of a sessile water drop on a dielectric substrate. In ac EW, small-amplitude oscillations of a drop differ from the Rayleigh linear modes of freely oscillating drops. In this paper, we demonstrate that changes in the time-averaged contact angle of the sessile drop attributed to the presence of an electric field and a solid substrate mainly caused this discrepancy. We combine the domain perturbation method with the Lindsted-Poincare method to derive an asymptotic formula for resonant frequency. Theoretical analysis shows that the resonant frequency is a function of the time-averaged contact angle. Each mode of the resonance frequency is a linear function of $\varepsilon_{1}$, which is the magnitude of the cosine of the time-averaged contact angle. The most dominant mode in this study, that is, the fundamental mode $n=2$, decreases linearly with $\varepsilon_{1}$. The results of the theoretical model are compared with those of both the experiments and numerical simulations. The average resonant frequency deviation between the perturbation solutions and numerical simulations is $4.3 \%$, whereas that between the perturbation solutions and the experiments is $1.8 \%$.
\end{abstract}

DOI: 10.1103/PhysRevE.90.033017

PACS number(s): 47.55.D-, 47.55.nd, 47.61.Fg, 68.05.-n

\section{INTRODUCTION}

The resonance frequency of a freely oscillating drop is known to be determined by mass and surface tension [1,2]. Meanwhile, drop viscosity dampens drop oscillations [3-5]. However, in the case of constrained drops, solid surface intervention generates an additional friction force, which modifies oscillation characteristics (e.g., resonance frequency or oscillating drop shape). Strani and Sabetta first studied the resonant frequencies of drops confined to a spherical bowl and found that constrained drops have an additional low-frequency mode [6,7]. Bostwick and Steen investigated the oscillation dynamics of a liquid drop pinned on a circle-of-contact [8]. They extended their studies to the linear oscillations of a spherical drop constrained by a spherical belt and obtained the frequency dependence on their constrained geometry $[9,10]$. Chang et al. [11] recently studied the resonance frequencies and oscillation modes of a sessile drop on a vertically oscillating plane and compared these measured resonance frequencies with theoretical predictions for unconstrained [1] and constrained drops $[9,10]$.

The aforementioned studies mainly focused on the oscillation dynamics of confined drops with a fixed contact line (CL). Voinov [12] and Cox [13] developed the Cox-Voinov law, a hydrodynamics theory, describing the changes in the dynamic contact angle caused by the viscous bending of the liquid-gas interface during CL movement. This law showed the dependence of the apparent contact angle, as well as the spreading rate, on the capillary number $(\mathrm{Ca}$, the relative strength of viscous forces relative to surface tension). Similarly, Hocking [14] suggested a linear relation between the CL velocity and contact angle deviation from the equilibrium value. Based on the Hocking conditions, Fayzrakhmanova and

\footnotetext{
${ }^{*}$ The first and the second author contributed equally to the paper. †hjhwang@ postech.ac.kr
}

Straube [15] studied the oscillation characteristics of a sessile drop. These characteristics include the stick slip dynamics, contact angle hysteresis (i.e., difference between advancing and receding contact angles), and frequency responses. Lyubimov et al. $[16,17]$ considered wetting parameters in developing a theoretical model of oscillating sessile drops with moving CLs and found that dissipation on moving CLs causes a shift in resonance frequency. Noblin et al. $[18,19]$ derived the resonant frequencies of the oscillation modes of sessile drops from pinned CLs to slipping CLs on a vertically vibrating plate using a combined dispersion relation of capillary-gravity waves and stationary surface wave conditions. Sharp and his corworkers [20] and Sharp [21] also derived a simple relation between resonance frequency and contact angle using the dispersion relation and standing wave states along the sessile drop profile length.

Mechanical vibration methods have difficulty in controlling CLs precisely and integrating the methods into the packaging frameworks of microelectronics in practical applications. Electrowetting (EW), a technique that electrically controls the wettability of a liquid sitting on a solid surface, is an alternative method for exploring the oscillation dynamics of a sessile drop [22] because electrical forces localized at the CL are controlled precisely. Moreover, ac EW (i.e., EW controlled by an ac electrical voltage) has many advantages: delay in contact-angle saturation $[23,24]$, decrease in contact-angle hysteresis $(\mathrm{CAH})$ [25], and prevention of sample adsorption onto solid surfaces $[26,27]$. These advantages enable the development of many practical applications in digital microfluidics, such as mixing enhancement [28-30] and drop transport without complicated electrical control [31,32].

A concrete understanding of the dynamics of drop oscillations induced by ac EW essentially improves the performance of microfluidic applications. Many studies about drop oscillation dynamics induced by ac EW have been reported [29,33-36]. Oh et al. [33] investigated ac frequency effects on oscillation behaviors, such as changes in shape modes and 
resonance frequencies. In their experiments, Lai et al. [34] observed phase differences between applied voltages and corresponding drop motions, as well as the beating phenomenon at resonance frequencies. Dash et al. [35] systematically studied ac frequency effects on shape modes and phase differences at different ac voltages. They also observed the sub-harmonic oscillation at half of the electrical forcing frequency. Based on both experiments and theoretical models proposed by Oh et al. [37], Hong et al. [36] explored the effects of drop viscosity on sessile drop resonance frequencies and oscillation amplitudes, in response to different ac voltages and frequencies.

In EW, the intervention of a solid surface may change the resonant frequency of sessile drop oscillations (e.g., sessile drop on a vertically vibrating plate). Thus, the oscillatory behavior of a drop in EW is expected to be different from that of a freely oscillating spherical drop. Oh et al. [33,37] derived a shape mode equation of drop oscillations in EW based on the conventional method for isolated spherical drops (bubbles) $[1,38]$ and the modified boundary conditions reflecting the effect of electrical stress concentrated on the CL. The shape mode equation successfully described the time evolution of the droplet shape in response to electrical stress. The $n$th mode resonant frequency in the shape mode equation is the dimensional form of the resonant frequency of a freely oscillating spherical drop [1]:

$$
\omega_{n}=\sqrt{n(n-1)(n+2) \frac{\rho R^{3}}{\gamma}},
$$

where $R, \rho$, and $\gamma$ are the volume-averaged droplet radius, droplet fluid density, and surface tension, respectively. However, the Rayleigh frequency Eq. (1) shows some discrepancy in predicting the resonance frequencies of drop oscillations by EW. Resonance frequencies from Eq. (1) are greater by about $12 \%$ than the experimental results $[33,34]$.

This study aims to identify an improved prediction method for the resonant frequency of drop oscillations induced by EW. We consider a mathematical model that includes the effect of contact angle changes through Young's angle or through electrical stress [37]. We consider two small parameters, $\varepsilon_{1}$ and $\varepsilon_{2}$, which measure distortion from the hemispherical sessile drop and the amplitude of drop oscillations in the conventional methods for an isolated spherical drop, respectively. We then expand the two parameters, combining the domain perturbation method and the Lindsted-Poincaré method [39,40]. This analysis provides an asymptotic formula for the dependence of the oscillating drop frequency on the time-averaged contact angle of a sessile drop. Fundamental resonant frequencies $(n=2)$ for various time-averaged contact angles are measured numerically and experimentally. Our numerical scheme is based on a level set method, and experiments are conducted under a typical EW system, where a tiny drop of water is placed on a dielectric layer surrounded by air. Resonant frequencies obtained from our theoretical analysis are compared with the numerical and experimental results.

\section{THEORETICAL ANALYSIS}

In this section, we formulate our problem analytically and conduct perturbation methods to study oscillating sessile drop

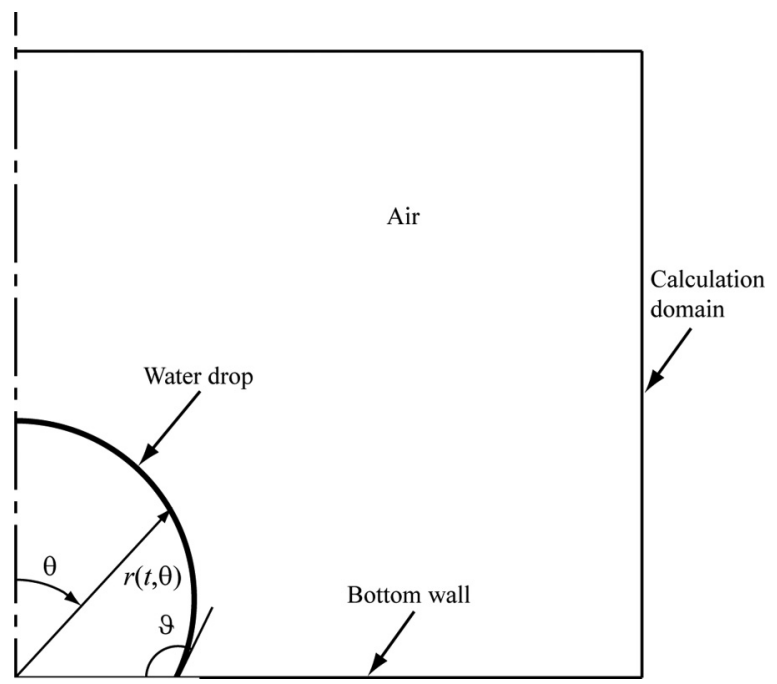

FIG. 1. A drop resting on a solid surface oscillates under the AC EW.

dynamics. We assume that the sessile drop fluid is inviscid, incompressible, and irrotational. The drop has volume of $\frac{2}{3} \pi R^{3}$, where $R$ is the sessile drop radius when the apparent contact angle is $90^{\circ}$. The droplet interface is described in spherical coordinates, where $r$ denotes the radial distance, and $\theta$ is the polar angle that varies between 0 and $\pi / 2$ because the drop rests on a solid. Inplane or plane-normal vibration may excite asymmetric shape modes. However, we assume that the oscillating drop shape is axisymmetric about $z$ axis because the effect of EW force appears uniformly on the sessile drop perimeter. Moreover, the CL motion and the instantaneous shapes of the interface are almost axially symmetric in our experiments. A problem sketch is provided in Fig. 1. Gravitational effects are not considered in our model because we consider the drop whose size is smaller than the capillary length for water $(2.7 \mathrm{~mm})$, which is a characteristic length scale representing the relative strength of gravity and surface tension. Given that the effect of viscosity on the resonant frequency of a small sessile water drop (approximately $5 \mu \mathrm{L}$ ) is negligible, our theoretical analysis results can be compared with the simulation in Sec. III and, experimental results in Sec. IV, respectively.

\section{A. Analytical problem formulation}

In this subsection, we establish an analytical formulation of the problem. The velocity potential $\psi$ that satisfies $\mathbf{u}=$ $\nabla \psi$ exists due to the assumption that the flow in the drop is irrotational. The velocity potential $\psi(r, \theta, t)$ and the shape function $F(\theta, t)$ are governed by the following equations:

$$
\begin{aligned}
\nabla^{2} \psi & =0, \\
\frac{\partial \psi}{\partial t}+\frac{1}{2}|\nabla \psi|^{2}+\frac{1}{\rho} p & =G(t), \\
\int_{0}^{\pi / 2} F^{3} \sin \theta d \theta & =1 .
\end{aligned}
$$


For even $n(n=2,4, \ldots)$,

$$
\begin{aligned}
& \int_{0}^{\pi / 2} \int_{0}^{2 \pi} F P_{n} \sin \theta \cos t d t d \theta=\frac{\pi R}{2 n+1} \varepsilon_{2}, \\
& \int_{0}^{\pi / 2} \int_{0}^{2 \pi} F P_{n} \sin \theta \sin t d t d \theta=0 .
\end{aligned}
$$

Equation (2) is the Laplace equation for velocity potential, whereas Eq. (3) is the Bernoulli equation for drop pressure $p(r, \theta, t)$, where $G(t)$ is an integration constant with respect to the spatial variables $r$ and $\theta$, which correspond to the continuity and the Navier-Stokes equation for velocity field $\mathbf{u}$ in Sec. III. Volume conservation of the drop provides the constraint Eq. (4). Equations (5) and (6) define the amplitude and phase of drop oscillations such that both are determined only by the term $P_{n}(\cos \theta) \cos t$ if the shape function is represented as a series of Legegendre polynomials [39]. $\varepsilon_{2}$ in Eq. (5) is a frequently used small parameter, which scales the oscillating motion amplitude in conventional methods for an isolated spherical drop.

On a solid substrate, the following impermeability condition should be satisfied:

$$
\frac{\partial \psi}{\partial \theta}=0 \quad \text { at } \quad \theta=\frac{\pi}{2} .
$$

The kinematic, the normal stress, and the tangential stress boundary conditions should be satisfied on the drop surface $[F(\theta, t)=r]$. The kinematic and the tangential stress conditions are given as:

$$
\begin{aligned}
(\nabla \psi \cdot \nabla)(r-F) & =-\frac{\partial(r-F)}{\partial t}, \\
\left\{\left[\mathbf{t} \cdot\left(\mathbf{n} \cdot \mathbf{T}^{h}\right)\right]\right\} & =0
\end{aligned}
$$

where $\{[\cdot]\}$ is defined as $[\cdot]_{\text {in }}-[\cdot]_{\text {out }}, \mathbf{t}$ is the unit tangential vector, and $\mathbf{n}$ is the outward unit normal vector at the interface.

In the EW-problem, the drop interface is deformed by wetting tension, which is the force acting on CL. This interface deformation results in macroscopic contact angle changes in EW [41]. In this paper, we modify the classical normal stress condition, which considers only the effect of the hydrodynamic stress $\mathbf{T}^{h}$, to normal stress condition to reflect drop deformation:

$$
\left\{\left[\mathbf{n} \cdot\left(\mathbf{n} \cdot \mathbf{T}^{h}\right)\right]\right\}+\gamma \cos \vartheta \frac{2}{R} \delta(\cos \theta)=\gamma(\nabla \cdot \mathbf{n}),
$$

where $\gamma$ is the surface tension, $\vartheta$ is the time-averaged sessile drop contact angle, and $\delta(\cos \theta)$ is the $\delta$ function, which approximates contact angle effect in the very narrow region of the CL.

The $\delta$ function can be decomposed into a series of Legendre polynomials as:

$$
\delta(\cos \theta)=\sum_{n=0}^{\infty} d_{n} P_{n}(\cos \theta),
$$

where

$$
d_{n}=\frac{2 n+1}{2} \int_{-1}^{1} \delta(\eta) P_{n}(\eta) d \eta=\frac{2 n+1}{2} P_{n}(0) .
$$

To obtain perturbation solutions, we introduce two independent small parameters. $\varepsilon_{1}$ represents the sessile drop quiescent deformation, which is determined by the time-averaged contact angle of the sessile drop as $\varepsilon_{1}=|\cos \vartheta| . \varepsilon_{2}$, the parameter in Eq. (5), scales the drop oscillation amplitude.

\section{B. Perturbation solution}

In this subsection, we solve Eqs. (2)-(6) with the boundary conditions from Eqs. (7)-(10) by using multiple parameter expansions. A new independent variable $\tau=\omega t$ is introduced. $\omega$ is initially an unspecified function of $\varepsilon_{1}$ and $\varepsilon_{2}$, despite acting as the drop oscillation frequency. Variable $t$ is the newly introduced variable $\tau$ different from the original time variable used in Sec. II A.

We seek approximate solutions for $\psi(r, \theta, t), F(\theta, t)$, and $\omega$ in a power series form in terms of $\varepsilon_{1}$ and $\varepsilon_{2}$ :

$$
f\left(r, \theta, t ; \varepsilon_{1}, \varepsilon_{2}\right)=\sum_{j=0}^{\infty} \sum_{k=0}^{\infty} \frac{\varepsilon_{1}^{j} \varepsilon_{2}^{k}}{j ! k !} f^{[j, k]}(r, \theta, t),
$$

where

$$
f^{[j, k]} \equiv\left(\frac{\partial}{\partial \varepsilon_{1}}+\frac{\partial F}{\partial \varepsilon_{1}} \frac{\partial}{\partial r}\right)^{j}\left(\frac{\partial}{\partial \varepsilon_{2}}+\frac{\partial F}{\partial \varepsilon_{2}} \frac{\partial}{\partial r}\right)^{k} f
$$

at $\varepsilon_{1}, \varepsilon_{2}=0$, and $r=R$.

Moreover, we define notation $f^{\langle j, k\rangle}$ as

$$
f^{\langle j, k\rangle} \equiv\left[\frac{\partial^{j+k} f}{\partial \varepsilon_{1}^{j} \partial \varepsilon_{2}^{k}}\right]_{\varepsilon_{1}=0, \varepsilon_{2}=0} .
$$

Given that the shape function is not a function of $r$, $F^{[j, k]}(\theta, t)=F^{\langle j, k\rangle}(\theta, t)$, and $F^{\langle j, k\rangle}$ at each order is assumed to be a series of Legendre polynomials:

$$
F^{\langle j, k\rangle}(\theta, t)=\sum_{n=0}^{\infty} a_{n}^{\langle j, k\rangle}(t) P_{n}(\cos \theta) .
$$

From the Laplace Eq. (2) with the natural boundary condition that the velocity at the drop center is bounded, the velocity potential is solved at each order in the form of

$$
\psi^{\langle j, k\rangle}(r, \theta, t)=\sum_{n=0}^{\infty} b_{n}^{\langle j, k\rangle}(t) r^{n} P_{n}(\cos \theta) .
$$

In $O(1,1)$, the sessile drop is a quiescent, hemispherical drop with contact angle $\vartheta=90^{\circ}$. The solutions are

$$
\psi^{\langle 0,0\rangle}=0, \quad \text { and } \quad F^{\langle 0,0\rangle}=R
$$

In $O\left(\varepsilon_{1}, 1\right)$, the time derivative and the velocity potential are zero because there are no oscillations, and the sessile drop is in equilibrium. Hence, the solutions are at steady states:

$$
\begin{aligned}
\psi^{\langle 1,0\rangle} & =0, \\
a_{n}^{\langle 1,0\rangle} & =\frac{\operatorname{sgn}(\mathrm{W})(2 \mathrm{n}+1)}{(n-1)(n+2)} R P_{n}(0),
\end{aligned}
$$

where $\operatorname{sgn}(W)$ indicates the sign of $\cos \vartheta$. 
In $O\left(1, \varepsilon_{2}\right)$, the solutions are equal to those in Rayleigh's free oscillation case:

$$
\begin{aligned}
a_{n}^{\langle 0,1\rangle} & =R \cos t, \text { for } n \text { even, } \\
\psi_{n}^{\langle 0,1\rangle} & =-\omega^{\langle 0,0\rangle} \frac{r^{n}}{n R^{n-1}} \sin t P_{n}(\cos \theta), \text { for } n \text { even, }
\end{aligned}
$$

and

$$
\omega_{n}^{\langle 0,0\rangle}=\sqrt{n(n-1)(n+2) \frac{\gamma}{\rho R^{3}}}, \text { for } n \text { even. }
$$

Owing to the volume conservation constraint Eq. (4), $a_{n}^{\langle 1,0\rangle}(t)$ and $a_{n}^{\langle 0,1\rangle}(t)$ should be zero for $n=0$ and odd $n$. Hence, only positive even modes $(n=2,4, \ldots)$ survive in Eqs. (20)-(23).

The correction term for the resonant frequency can be obtained by deriving the shape mode equation at the order of $O\left(\varepsilon_{1}, \varepsilon_{2}\right)$. A secular term in the shape mode equation appears for $m=n$ :

$$
\begin{aligned}
& \ddot{a}_{m}^{\langle 1,1\rangle}(t)+\frac{m(m+1)(m-1)(m+2)}{n(n+1)(n-1)(n+2)} a_{m}^{\langle 1,1\rangle}(t) \\
& =\frac{(2 m+1)}{2}\left[\frac{\omega_{m}^{\langle 1,0\rangle}}{\omega_{n}^{\langle 0,0\rangle}}\left(1+\frac{m}{n}\right)\left\langle P_{n}, P_{m}\right\rangle\right. \\
& \quad+\operatorname{sgn}(W) \frac{(2 n+1) P_{n}(0)}{(n-1)(n+2)} \\
& \left.\quad \times\left\langle(m-n+1) P_{n}^{2}+\frac{1}{n}\left(\frac{\partial P_{n}}{\partial \theta}\right)^{2}, P_{m}\right\rangle\right] \cos t
\end{aligned}
$$

To remove the secular term, $\omega_{n}^{\langle 1,0\rangle}$ should satisfy the following:

$$
\begin{aligned}
\frac{\omega_{n}^{\langle 1,0\rangle}}{\omega_{n}^{\langle 0,0\rangle}}= & -\operatorname{sgn}(W) \frac{(2 n+1)^{2} P_{n}(0)}{4(n-1)(n+2)} \\
& \times\left\langle P_{n}^{2}+\frac{1}{n}\left(\frac{\partial P_{n}}{\partial \theta}\right)^{2}, P_{n}\right\rangle,
\end{aligned}
$$

where $\langle h, g\rangle$ is the inner product

$$
\langle h(\theta), g(\theta)\rangle=\int_{0}^{\pi} h(\theta) g(\theta) \sin \theta d \theta .
$$

Hereafter, the resonant frequency $\omega_{n}$ means the form including the correction term of order $\varepsilon_{1}$ as follows:

$$
\omega_{n}=\omega_{n}^{\langle 0,0\rangle}+\varepsilon_{1} \omega_{n}^{\langle 1,0\rangle} .
$$

\section{NUMERICAL ANALYSIS}

In this section, we study the oscillating sessile drop dynamics by using a numerical model based on a level set method. A $5-\mu \mathrm{L}$ volume of water drop rests on a surface with an undisturbed contact angle of $116^{\circ}$. The computational domain is $4 \mathrm{~mm} \times 4 \mathrm{~mm}$ and is evenly discretized into $200 \times 200$ square cells. Both the water drop and its surrounding air are incompressible and immiscible. Navier's slip boundary condition is applied to the bottom wall, whereas no-slip boundary condition is applied to other boundaries. We use OpenFOAM, an open-source $\mathrm{C}++$ library for complex physics simulations based on the finite volume method, to solve the fluid flow [42]. Velocity $\mathbf{u}$ and pressure $p$ are obtained from the incompressible one-fluid formulation of the continuity and the Navier-Stokes equation,

$$
\begin{aligned}
\nabla \cdot \mathbf{u} & =0, \\
\rho\left[\frac{\partial \mathbf{u}}{\partial t}+(\mathbf{u} \cdot \nabla) \mathbf{u}\right]+\nabla p & =\mu \nabla^{2} \mathbf{u}+\mathbf{F}_{s},
\end{aligned}
$$

where $\rho$ and $\mu$ are the density and viscosity, respectively.

$\mathbf{F}_{s}$ is the body force arising from surface tension $\gamma$ and is given as:

$$
\mathbf{F}_{s}=-\gamma(\nabla \cdot \mathbf{n}) \nabla \Phi_{\mathrm{lsf}},
$$

where $\Phi_{\text {lsf }}$ denotes the level set function, and $\mathbf{n}$ is the outward unit normal vector.

Parameter values are given as $\rho_{\text {water }}=1000 \mathrm{~kg} / \mathrm{m}^{3}, \rho_{\text {air }}=$ $1.25 \mathrm{~kg} / \mathrm{m}^{3}, \mu_{\text {water }}=1 \times 10^{-3} \mathrm{~Pa} \mathrm{~s}, \mu_{\text {air }}=1.82 \times 10^{-5} \mathrm{~Pa} \mathrm{~s}$, and $\gamma=7.2 \times 10^{-2} \mathrm{~N} / \mathrm{m}$, in accordance with the aqueous drop described in Sec. IV. Subscript water and air indicate the water drop and surrounding air, respectively.

Navier's slip boundary condition [43] is applied to the entire bottom wall to avoid the stress singularity problem at the CL as

$$
F_{t}=\frac{\mu}{l_{s}} \mathbf{u}_{t, \text { wall }},
$$

where $F_{t}$ is the tangential component of stress acting on a cell in contact with the bottom wall, and $\mathbf{u}_{t, \text { wall }}$ is the tangential component of the flow velocity at the bottom wall. $l_{s}$ is the slip length that defines the distance below the bottom surface at which the extrapolated fluid velocity reaches zero, and is set to half of the mesh size. To capture the moving interface, our own code is added to OpenFOAM by using a modified conservative level set method [44]. The interface is implicitly defined as $\Phi_{\text {lsf }}=\frac{1}{2}$, which is initially set to $\Phi_{\text {lsf }, 0}=\frac{1}{1+\exp (-x / l)}$, where $x$ indicates the signed distance from the interface, and $\iota$ is the parameter that determines numerical interface thickness $[45,46] . \iota$ is set as the mesh size in this paper. The interface moves along with the neighboring fluid, which is described by the advection equation as:

$$
\frac{\partial \Phi_{\mathrm{lsf}}}{\partial t}+\nabla \cdot\left(\Phi_{\mathrm{lsf}} \mathbf{u}\right)=0 .
$$

The reinitialization process is performed after each time step to maintain the initial smooth profile of $\Phi_{\text {lsf }}$ across the interface.

$$
\frac{\Phi_{\mathrm{lsf}}}{\partial \tau}+\nabla \cdot\left[\Phi_{\mathrm{lsf}}\left(1-\Phi_{\mathrm{lsf}}\right) \mathbf{n}\right]=\iota \nabla \cdot\left(\nabla \Phi_{\mathrm{lsf}}\right),
$$

where $\tau$ indicates an artificial time used only in intermediate reinitialization. The advection and reinitialization steps are performed in the conservative scheme [45] to prevent the loss or gain of mass.

Electric force at the $\mathrm{CL}$ is numerically implemented by apparent contact angle changes following the LippmannYoung equation.

$$
\gamma \cos \vartheta=\frac{\epsilon_{d} V^{2}}{2 d}+\gamma \cos \theta_{Y},
$$

where $\epsilon_{d}$ is the dielectric constant of the insulator, $V$ is the applied voltage, $d$ is the insulator thickness, $\theta_{Y}$ is Young's angle (representing the contact angle without externally 

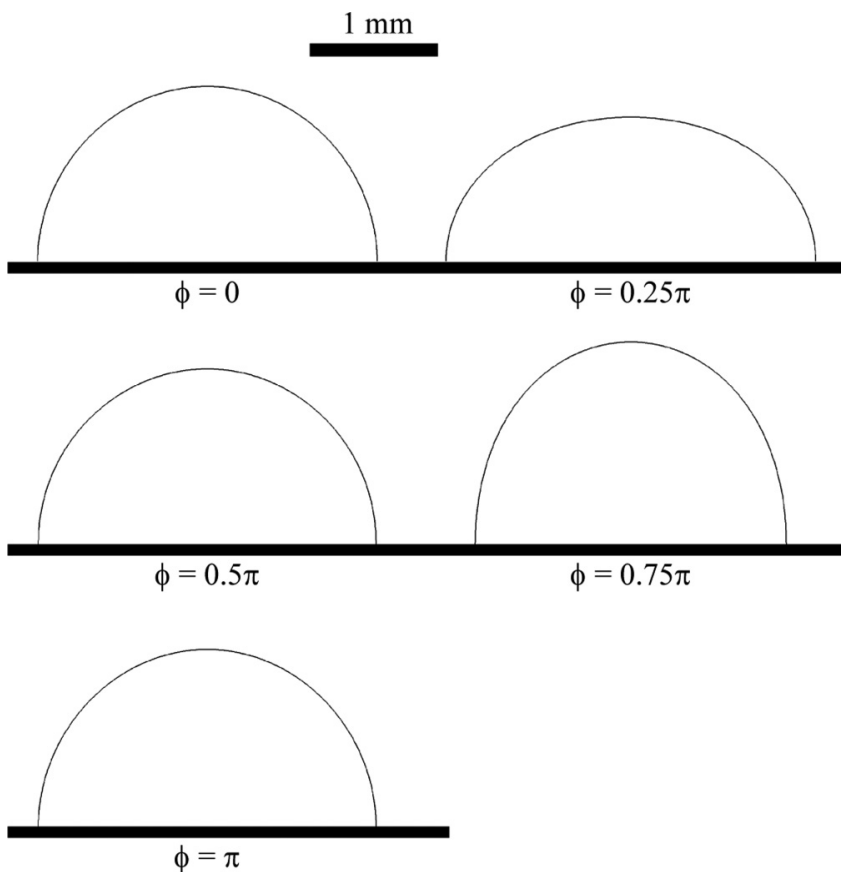

FIG. 2. Numerically obtained instantaneous shapes of a drop with average contact angle of $90^{\circ}$ during one period of oscillation.

applied electrical potential), and $\vartheta$ is the apparent contact angle of the sessile drop. Microscopic contact angle $\theta_{Y}$ is assumed to be undisturbed by electrical stress in EW [41]. We set Young's angle $\theta_{Y}$ as $116^{\circ}$ according to our experimental setup. The Lippmann-Young equation accurately predicts the equilibrium contact angle $\vartheta$ until contact angle saturation occurs [22]. We call the right-hand side of Eq. (34) wetting tension $W$, which represents the net force per unit length, driving wetting of a drop. The wetting tension is distributed at the surface in a very narrow region near the CL.

Figure 2 shows instantaneous images of the sessile drop at $\phi=0, \pi / 4, \pi / 2,3 \pi / 4$, and $\pi$ with time-averaged contact angle $\vartheta=90^{\circ}$, where $\phi$ denotes the oscillation phase angle. Figure 3 shows the oscillation patterns for different timeaveraged contact angles. Oscillation patterns are obtained by superposing instantaneous images. The time-averaged contact angles are (a) $70^{\circ}$, (b) $80^{\circ}$, (c) $90^{\circ}$, (d) $100^{\circ}$, (e) $110^{\circ}$, and (f) $120^{\circ}$. All numerical results in this paper were measured in the 10th period of the drop oscillations. Oscillation amplitudes were calculated as the maximum change of drop base diameter during one period. Frequency was scanned with a resolution of $0.5 \mathrm{~Hz}$ to identify the resonant frequencies.

We then compared our fixed contact angle model with dynamic contact angle models, such as the Hocking condition or Cox-Voinov relation.

The Hocking condition is

$$
\gamma \cos \vartheta_{d}=\gamma \cos \vartheta-\lambda U
$$

where $\vartheta_{d}, \lambda$, and $U$ are the dynamic contact angle, friction coefficient, and CL velocity of the sessile drop, respectively.

The Cox-Voinov relation is given by

$$
\vartheta_{d}^{3}=\vartheta^{3}+C_{\mathrm{cv}} C a,
$$
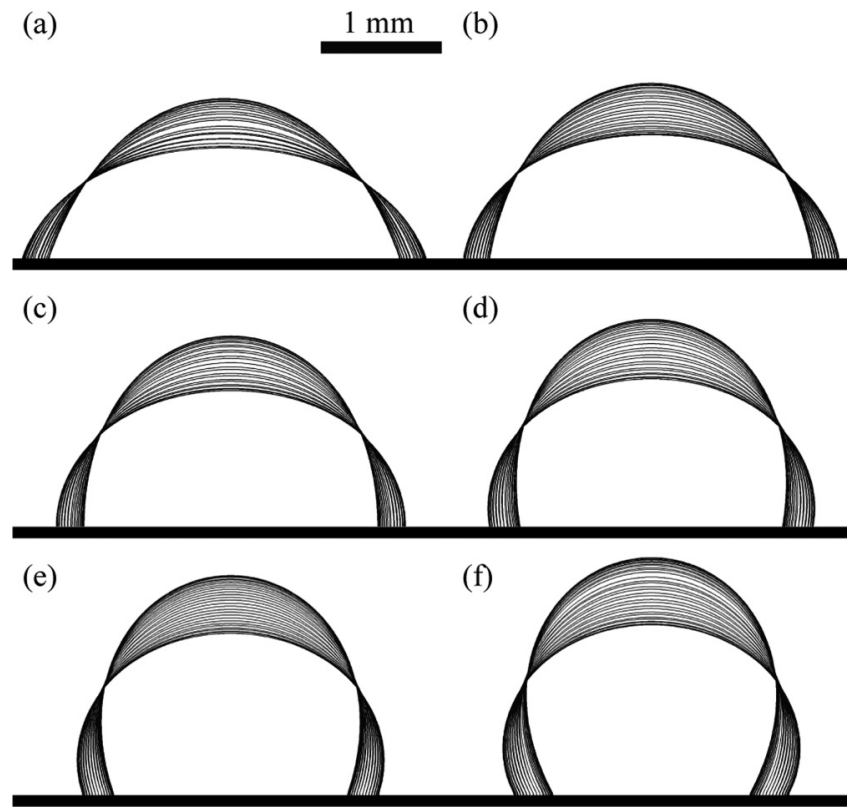

FIG. 3. Oscillation patterns obtained by superposing numerical drop shapes for the average contact angle of (a) $70^{\circ}$, (b) $80^{\circ}$, (c) $90^{\circ}$, (d) $100^{\circ}$, (e) $110^{\circ}$, and (f) $120^{\circ}$.

where $C a$ and $C_{\mathrm{cv}}$ are the capillary number and Cox-Voinov relation coefficient, respectively.

By combining our numerical method with the dynamic contact angle models as a CL boundary condition, we obtained the sessile drop resonant frequencies for the contact angle $\vartheta=90^{\circ}$. The coefficients for the Hocking condition and Cox-Voinov relations agreed with those derived from the experimental results (e.g., $\lambda=0.05$ for the Hocking condition and $C_{\mathrm{cv}}=382$ for the Cox-Voinov relation). The corresponding resonant frequencies are 38,38 , and $38.5 \mathrm{~Hz}$ for the Hocking condition, Cox-Voinov relations, and fixed contact angle models, respectively. Resonant frequencies are almost the same regardless of contact angle models. For this reason, we did not consider the dynamic contact angle models in the present paper.

\section{EXPERIMENT}

In this section, we describe the experiments conducted to validate our theoretical model. The experimental setup employed to observe drop oscillations is similar to a typical apparatus for EW experiments [33,37], as shown in Fig. 4. An aqueous drop of $0.1 \mathrm{M} \mathrm{NaCl}$ was placed on a bottom electrode with a micropipette. Volume of the conducting drop was consistently fixed at $5 \mu \mathrm{L}$ corresponding to the initial base radius of approximately $1 \mathrm{~mm}$. The undisturbed water drop contact angle without an externally applied electrical potential, so-called Young's angle, was approximately $116^{\circ}$. The bottom electrode was indium tin oxide (ITO) electrode plate coated with a dielectric layer (Parylene-C) of 5- $\mu \mathrm{m}$ thickness and a hydrophobic layer (DuPont, AF1600) of $100 \mathrm{~nm}$. A stainless steel wire with $80-\mu \mathrm{m}$ diameter was used as a top electrode. Electrical signals were produced by a function generator (33220A, Agilent) and amplified 100 times 


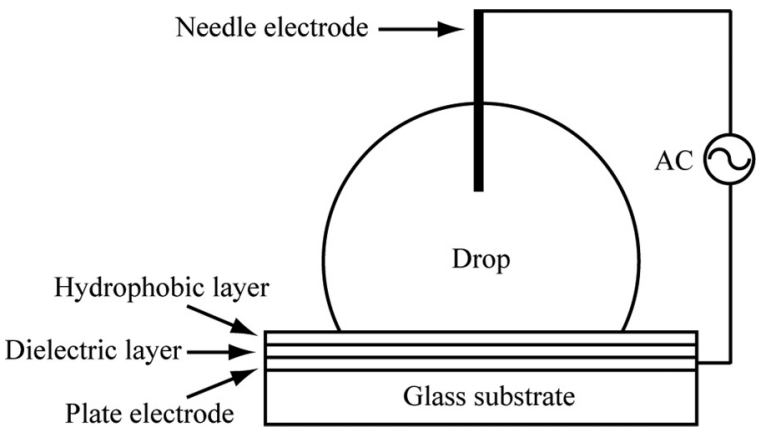
EW.

FIG. 4. Experimental setup for an oscillating drop driven by AC

by a voltage amplifier (A800, FLC Electronics). Contact angles were measured using a contact angle goniometer (SmartDrop, Femtofab). The temporal evolution of drop oscillations was recorded consecutively with a high speed camera (Fastcam SA3, Photron) at a frame rate of $1000 \mathrm{fps}$.

The drop contact angle and oscillation amplitude were controlled by manipulating the electrical signal. The periodic electrical signal was combined with DC signals to change the average apparent contact angle and AC signals to perturb the drop oscillations. Figure 5 shows (a) the electrical signal

(a)

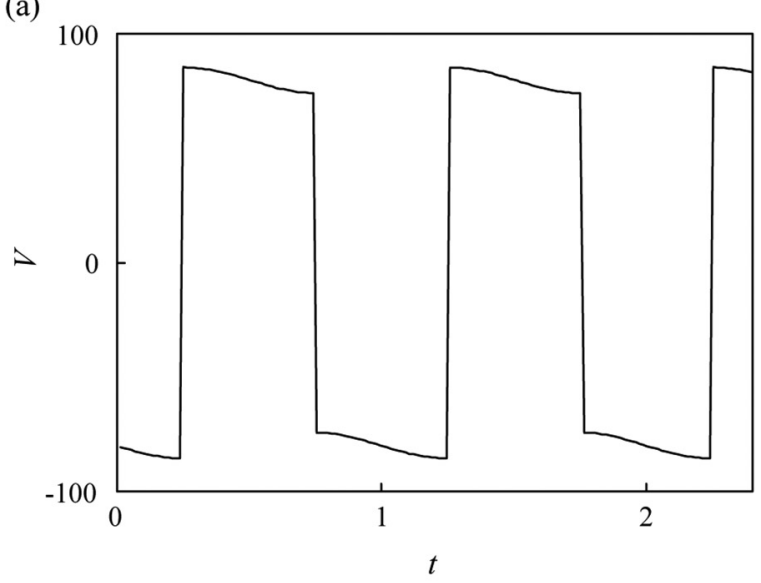

(b)

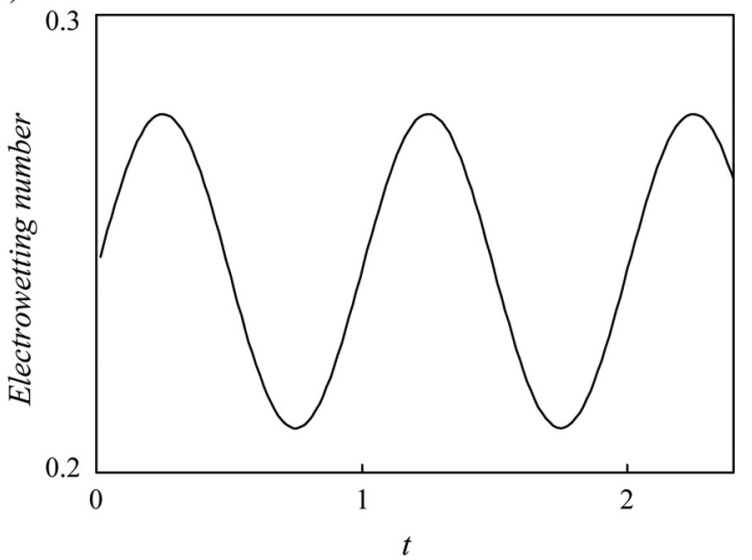

FIG. 5. (a) An electrical signal combined with DC and AC components as a function of time scaled with one period of signal and (b) the corresponding EW number $\eta$.

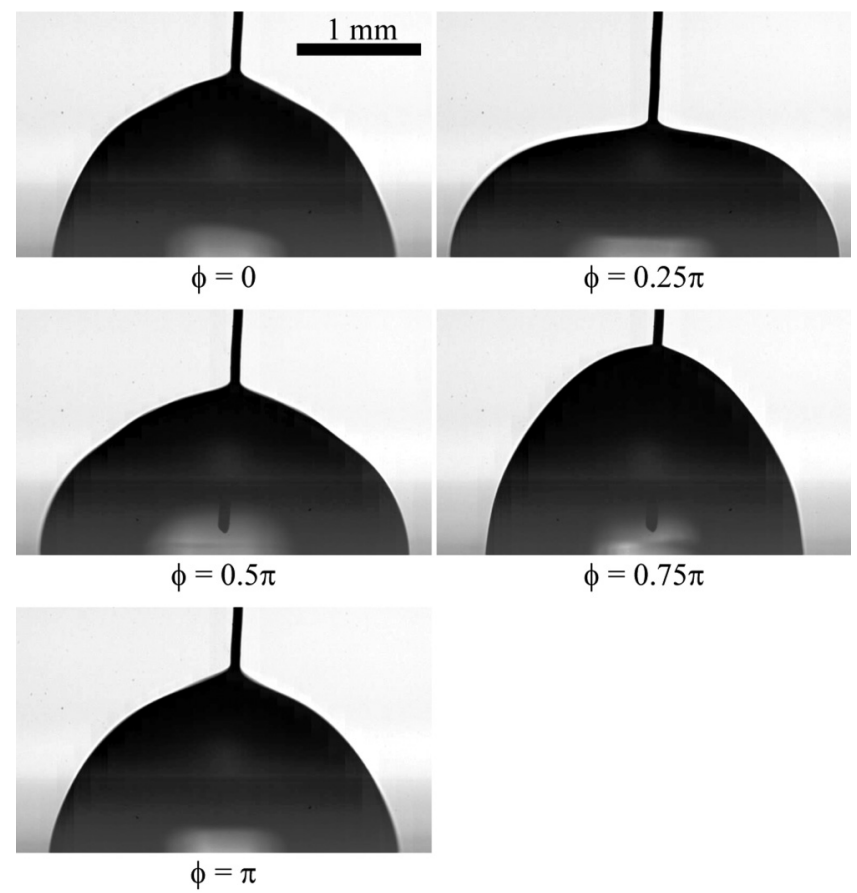

FIG. 6. Experimentally captured instantaneous images of the drop with the average contact angle of $88^{\circ}$ during one period of oscillations.

obtaining the time-averaged contact angle $\vartheta=103^{\circ}$ and (b) the corresponding EW number $\eta$. The dc voltage and the amplitude for the ac field are 80 and $5.44 \mathrm{~V}$, respectively. The dimensionless EW number is defined as the ratio of the electrical force to the surface tension as follows:

$$
\eta=\epsilon_{d} V(t)^{2} / 2 d \gamma
$$

where $\epsilon_{d}$ is the insulator dielectric constant, $V(t)$ is the applied voltage, $d$ is the insulator thickness, and $\gamma$ is the surface tension.

Figure 6 shows the instantaneous images of the oscillating drop. The instantaneous images are captured at $\phi=0, \pi / 4$, $\pi / 2,3 \pi / 4$, and $\pi$ with the time-averaged contact angle $\vartheta=$ $88^{\circ}$, where $\phi$ denotes the input voltage phase angle.

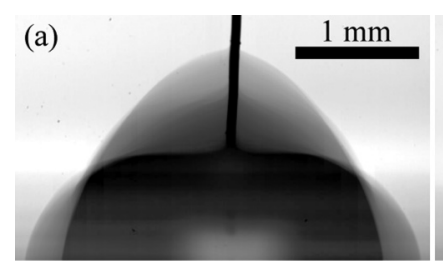

(b)

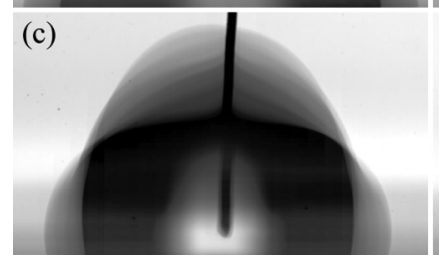

(d)

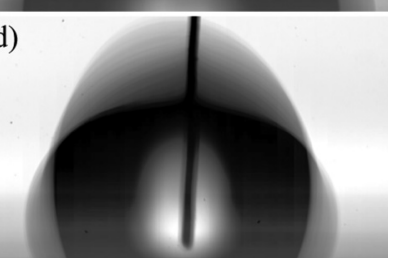

FIG. 7. Oscillation patterns obtained by superposing experimentally captured drop images for the average apparent contact angle of (a) $83^{\circ}$, (b) $88^{\circ}$, (c) $103^{\circ}$, and (d) $115^{\circ}$. 
Figure 7 shows the oscillation patterns for different timeaveraged contact angles, which were obtained by superposing experimentally captured instantaneous images. Time-averaged contact angles are changed to (a) $83^{\circ}$, (b) $88^{\circ}$, (c) $103^{\circ}$, and (d) $115^{\circ}$ by altering dc signal levels. The ac signal amplitude was controlled to have the same oscillation amplitude for each contact angle at the resonant frequency of approximately $0.6 \mathrm{~mm}$. Oscillation amplitude was calculated as the maximum change in drop base diameter during one period.

\section{RESULTS AND DISCUSSION}

In this section, we first show that the theoretical model proposed in Sec. II is valid for the usual range of the contact angle $\left(70^{\circ}\right.$ to $\left.120^{\circ}\right)$, and then compare the resonance frequencies obtained by the theoretical model with those obtained by both the experiments and numerical simulations.

We checked whether the contact angle $\bar{\vartheta}$ obtained from the perturbation solution was consistent with the original contact angle $\vartheta$ to validate our theoretical model. Given that the sessile drop amplitude in equilibrium is approximated by $a_{n}^{\langle 1,0\rangle}$ in Eq. (20), the shape function at equilibrium is approximated as follows:

$$
\begin{aligned}
\bar{F}(\theta, t) \approx & R\left[1+\operatorname{sgn}(W) \varepsilon_{1} \sum_{n=2}^{\infty} \frac{2 n+1}{(n-1)(n+2)}\right. \\
& \left.\times P_{n}(0) P_{n}(\cos \theta)\right],
\end{aligned}
$$

where $\operatorname{sgn}(W) \varepsilon_{1}$ is determined by $\operatorname{sgn}(W) \varepsilon_{1}=\cos \vartheta$.

The sessile drop apparent contact angle can be approximated by the perturbation solution of the shape function (38) as

$$
\begin{aligned}
\cos \bar{\vartheta}= & \left.\mathbf{t} \cdot \mathbf{e}_{x}\right|_{\theta=89^{\circ}}=\frac{1}{\left|\nabla\left(r_{b}-\bar{F}\right)\right|}\left[-\frac{1}{r_{b}} \frac{\partial\left(r_{b}-\bar{F}\right)}{\partial \theta} \sin \theta\right. \\
& \left.+\frac{\partial\left(r_{b}-\bar{F}\right)}{\partial r} \cos \theta\right]\left.\right|_{\theta=89^{\circ}},
\end{aligned}
$$

where $\mathbf{t}$ is the unit tangent vector of the drop surface at the $\mathrm{CL}, \mathbf{e}_{x}$ is the standard vector in Cartesian coordinates, and $r_{b}$ is the base radius of oscillating sessile drops at equilibrium. We numerically calculated the dimensionless base radii $r_{b}$ and then nondimensionalized the base radii according to the hemispherical drop radius. The dimensionless radii are 1.18, $1.09,1,0.91,0.82$, and 0.73 for contact angles $70^{\circ}, 80^{\circ}, 90^{\circ}$, $100^{\circ}, 110^{\circ}$, and $120^{\circ}$, respectively. The right-hand side of Eq. (39) is always zero at $\theta=90^{\circ}$ because the perturbation solution $\bar{F}$ is a weighted sum of $P_{2 n}(\cos \theta)$. Hence, we used the slight deviation $\theta=89^{\circ}$ from $\theta=90^{\circ}$ to approximate the apparent contact angle.

The contact angle value $\bar{\vartheta}$ from Eq. (39) and the original contact angle $\vartheta$ for various values of $\cos \vartheta$ are compared in Fig. 8. The solid line represents the original contact angle $\vartheta$, and the markers represent $\bar{\vartheta}$ from Eq. (39). The shape mode is used up to $N=90$ to compute the perturbation solution $\bar{F}$. Good agreement was observed in the range of $\vartheta=70^{\circ}$ to $\vartheta=120^{\circ}$. Although the deviation between two data points becomes larger as the apparent contact angle $\vartheta$ becomes farther from $90^{\circ}$, the deviation is less than $5 \%$ in the given range. In

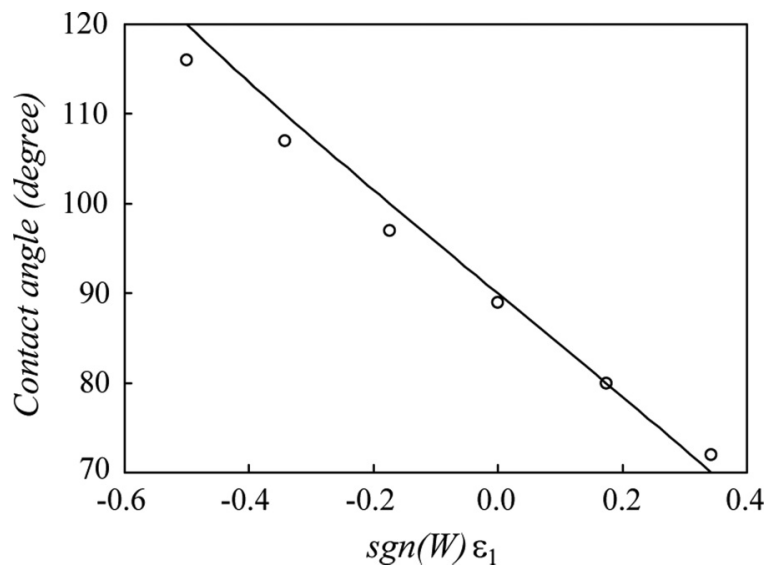

FIG. 8. Comparison between the original contact angle (solid line) and the approximation for the contact angle from Eq. (38) (markers).

EW applications, however, the contact angle of electrowetted drops on a hydrophobic surface is usually limited in the range of $70^{\circ}$ to $120^{\circ}$ because of the emergence of contact-angle saturation [37]. Thus, the present method which is based on the assumption that the drop shape is not significantly distorted from the hemispherical shape $\left(\vartheta=90^{\circ}\right)$ could be valid for most practical situations in EW.

Resonance frequency is known to decrease as the apparent contact angle $\vartheta$ increases. This result could not be predicted by Rayleigh's linear mode [1,33,37]. By the asymptotic Eqs. (25) and (27), we can explicitly observe the dependence of resonant frequency on contact angle:

$$
\omega_{n}=\omega_{n}^{\langle 0,0\rangle}+\omega_{n}^{\langle 1,0\rangle} \varepsilon_{1}=\omega_{n}^{\langle 0,0\rangle}+f(n) \cos \vartheta,
$$

where $\varepsilon_{1}=|\cos \vartheta|$ and $f(n)=\operatorname{sgn}(W) \omega_{n}^{\langle 1,0\rangle}$.

Note that $\omega_{n}$ in Eq. (40) decreases for $n=2,6,10, \ldots$ as contact angle $\vartheta$ increases in the range of $70^{\circ}$ to $120^{\circ}$.

We focused on the resonance frequencies of the fundamental $P_{2}$ mode in this paper because the fundamental mode $P_{2}$ dominated the drop oscillation, and the other shape modes were observed to be smaller than those at the $P_{2}$ mode [33]. The dominant effect of the $P_{2}$ mode was also observed in our theoretical model, following (25). The magnitudes of $\omega_{n}^{\langle 1,0\rangle} / \omega_{n}^{\langle 0,0\rangle}$ for $n=2,4,6$, and 8 are $0.2232,0.0531,0.0257$, and 0.0197 , respectively.

The fundamental mode $(n=2)$ of the resonance frequency predicted by the asymptotic solution Eq. (27) was compared to those modes from numerical simulations and experiments in Fig. 9 for various values of time-averaged contact angles. For asymptotic results, the parameter values, except for the liquid drop viscosity, were equivalent to those in the numerical simulations and experiments. The drop was assumed inviscid in the asymptotic case because the viscosity effect on the resonant frequency of a small sessile drop (about $5 \mu \mathrm{l}$ ) is negligible [33,47]. The $P_{2}$ shape mode resonant frequency was determined as the frequency with the largest oscillation amplitude in the numerical simulation. In experiments, the resonant frequencies for the contact angles in Fig. 7 were measured by scanning the frequency with a $0.5-\mathrm{Hz}$ resolution. Electrical signal frequency was scanned near the $P_{2}$ shape 


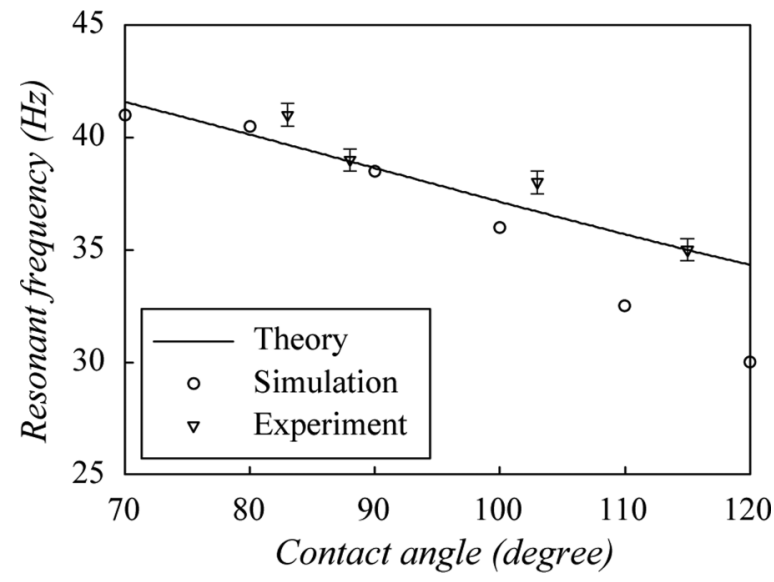

FIG. 9. Comparison between resonant frequencies obtained by theory, simulation, and experiment as a function of contact angle.

mode resonant frequency of the drop. Resonant frequency was determined as the frequency at which the largest oscillation amplitude of drop base diameter occurred.

Figure 9 shows that the frequencies obtained by the perturbation method are close to those from numerical simulations and experiments. All $P_{2}$ mode frequencies of 5- $\mu \mathrm{L}$ aqueous drop obtained from experiments, numerical simulations, and asymptotic solutions were very close to $38.5 \mathrm{~Hz}$ at $\vartheta=90^{\circ}$, corresponding to the Rayleigh resonant frequency in Eq. (1). The three results deviated from the Rayleigh frequency as the contact angle deviation from $90^{\circ}$ increased. However, the results of all three cases consistently show that resonance frequency decreases as contact angle $\vartheta$ increases. The average deviation of resonant frequency between the perturbation solutions and numerical simulations is $4.3 \%$, whereas that between the perturbation solutions and the experiments is $1.8 \%$.

\section{CONCLUSION}

We investigated the effects of time-averaged contact angles on resonant frequencies of an oscillating sessile drop on a dielectric substrate driven by AC EW. The correction term of the resonance frequency was obtained when the asymptotic analysis was extended to $O\left(\varepsilon_{1}, \varepsilon_{2}\right)$. The asymptotic solution showed the decrease in resonant frequencies with increasing contact angles: this result could not be predicted in the Rayleigh mode $[1,33,37]$. The contributions of the capillary force and electric force to the resonant frequency are shown explicitly since the contact angle effect is interpreted as a wetting tension on the CL of the sessile drop through the Lippmann-Young equation. The resonant frequencies of $\mathrm{AC}$ EW-induced sessile drop oscillations are measured through experiments and numerical simulations based on the level set method. The resonant frequencies obtained by the asymptotic analysis agree with the numerical and experimental results within the contact angle range $70^{\circ}$ to $120^{\circ}$.

\section{ACKNOWLEDGMENTS}

Hyung Ju Hwang was partly supported by the Basic Science Research Program (Grants No. 2010-0008127 and
No. 2013053914) by the National Research Foundation of Korea (NRF). This work was also supported by an NRF grant funded by the Korean government (MSIP) (Grant No. 2008-0061991). The authors appreciate the assistance of $\mathrm{Mr}$. Youngmin $\mathrm{Oh}$ in the preparation of this manuscript.

\section{APPENDIX A: $O\left(\varepsilon_{1}, 1\right)$}

Given that $\varepsilon_{2}=0$, only a quiescent deformation of the drop surface is observed. The time derivative and the velocity potential disappear.

$$
\begin{aligned}
\psi^{\langle 1,0\rangle} & =0, \\
\frac{1}{\rho} p^{\langle 1,0\rangle} & =0 .
\end{aligned}
$$

Given that both $F^{\langle 0,0\rangle}$ and $F^{\langle 1,0\rangle}$ are steady, the kinematic boundary condition always holds:

$$
-\omega^{\langle 0,0\rangle} \frac{\partial F^{\langle 1,0\rangle}}{\partial t}-\omega^{\langle 1,0\rangle} \frac{\partial F^{\langle 0,0\rangle}}{\partial t} \equiv 0 .
$$

The normal boundary condition becomes

$$
\begin{gathered}
p^{\langle 1,0\rangle}+\operatorname{sgn}(W) \frac{2 \gamma}{R} \frac{2 n+1}{2} P_{n}(0) P_{n}(\cos \theta) \\
=\frac{\gamma}{R^{2}}(n-1)(n+2) a_{n}^{\langle 1,0\rangle} P_{n}(\cos \theta),
\end{gathered}
$$

where $\operatorname{sgn}(W)$ is the $\operatorname{sign}$ of $\cos \vartheta$, which is equal to that of the wetting tension $W$.

$$
a_{n}^{\langle 1,0\rangle}=\frac{\operatorname{sgn}(\mathrm{W})(2 \mathrm{n}+1)}{(n-1)(n+2)} R P_{n}(0) .
$$

APPENDIX B: $O\left(1, \varepsilon_{2}\right)$

$$
\nabla^{2} \psi^{\langle 0,1\rangle}=0
$$

with

$$
\begin{array}{r}
\frac{\partial \psi^{\langle 0,1\rangle}}{\partial r} \neq \infty(r=0), \\
\omega^{\langle 0,0\rangle} \frac{\partial \psi^{\langle 0,1\rangle}}{\partial t}+\omega^{\langle 0,1\rangle} \frac{\partial \psi^{\langle 0,0\rangle}}{\partial t}+\frac{1}{\rho} p^{\langle 0,1\rangle}=0 .
\end{array}
$$

In $O\left(1, \varepsilon_{2}\right), \psi^{[0,1]}=\psi^{\langle 0,1\rangle}$ and $p^{[0,1]}=p^{\langle 0,1\rangle}$.

On the interface $r=F(\theta, t)$, we obtain

$$
\begin{aligned}
\frac{\partial \psi^{\langle 0,1\rangle}}{\partial r} & =\omega^{\langle 0,0\rangle} \frac{\partial F^{\langle 0,1\rangle}}{\partial t}+\omega^{\langle 0,1\rangle} \frac{\partial F^{\langle 0,0\rangle}}{\partial t}, \\
p^{\langle 0,1\rangle} & =\frac{\gamma}{R^{2}} \sum(n-1)(n+2) a_{n}^{\langle 1,0\rangle} P_{n}(\cos \theta) .
\end{aligned}
$$

Based on the above equations:

$$
\begin{gathered}
\psi^{\langle 0,1\rangle}=\sum b_{n}^{\langle 0,1\rangle} r^{n} P_{n}(\cos \theta), \\
\sum \omega_{n}^{\langle 0,0\rangle} \dot{b}_{n}^{\langle 0,1\rangle}(t) r^{n} P_{n}(\cos \theta)=-\frac{1}{\rho} p^{\langle 0,1\rangle}, \\
\sum n b_{n}^{\langle 0,1\rangle}(t) R^{n-1} P_{n}(\cos \theta)=\sum \omega_{n}^{\langle 0,0\rangle} \dot{a}_{n}^{\langle 0,1\rangle}(t) P_{n}(\cos \theta),
\end{gathered}
$$


or

$$
b_{n}^{\langle 0,1\rangle}(t)=\omega_{n}^{\langle 0,0\rangle} \frac{R^{1-n}}{n} \dot{a}_{n}^{\langle 0,1\rangle}(t) .
$$

If we substitute Eq. (B7) into Eq. (B5), then

$$
-\rho \omega_{n}^{\langle 0,0\rangle}{\dot{b_{n}}}^{\langle 0,1\rangle}(t) r^{n}=\frac{\gamma}{R^{2}}(n-1)(n+2) a_{n}^{\langle 0,1\rangle}(t) .
$$

Finally, we obtain the second-order ODE for $a_{n}^{\langle 0,1\rangle}(t)$ :

$$
\ddot{a}_{n}^{\langle 0,1\rangle}(t)+\frac{n(n-1)(n+2) \gamma}{\left(\omega_{n}^{\langle 0,0\rangle}\right)^{2} \rho R^{3}} a_{n}^{\langle 0,1\rangle}(t)=0 .
$$

Thus, we obtain

$$
a_{n}^{\langle 0,1\rangle}=\cos t
$$

and

$$
\omega_{n}^{\langle 0,0\rangle}=\sqrt{n(n-1)(n+2) \frac{\gamma}{\rho R^{3}}} .
$$

\section{APPENDIX C: $O\left(\varepsilon_{1}, \varepsilon_{2}\right)$}

In this case, we obtain

$$
\psi^{\langle 1,1\rangle}=0
$$

with

$$
\frac{\partial \psi^{\langle 1,1\rangle}}{\partial r} \neq \infty \quad(r=0) .
$$

Thus,

$$
\begin{gathered}
\psi^{\langle 1,1\rangle}=\sum b_{n}^{\langle 1,1\rangle}(t) r^{n} P_{n}(\cos \theta), \\
\omega^{\langle 0,0\rangle} \frac{\partial \psi^{\langle 1,1\rangle}}{\partial t}+\omega^{\langle 1,0\rangle} \frac{\partial \psi^{\langle 0,1\rangle}}{\partial t}+\frac{1}{\rho} p^{\langle 1,1\rangle}=0 .
\end{gathered}
$$

In $O\left(\varepsilon_{1}, \varepsilon_{2}\right)$,

$$
f^{[1,1]}=\left[f^{\langle 1,1\rangle}+F^{\langle 1,0\rangle} \frac{\partial f^{\langle 0,1\rangle}}{\partial r}\right]_{r=R},
$$

where $f$ is the velocity potential $\psi$ or the pressure $p$.
On the interface, $r=F(\theta, t)$ :

$$
\begin{aligned}
\frac{\partial \psi^{[1,1]}}{\partial r}= & {\left[\frac{\partial \psi^{\langle 1,1\rangle}}{\partial r}+F^{\langle 1,0\rangle} \frac{\partial^{2} \psi^{\langle 0,1\rangle}}{\partial r^{2}}\right]_{r=R} } \\
= & {\left[\omega^{\langle 0,0\rangle} \frac{\partial F^{\langle 1,1\rangle}}{\partial t}+\omega^{\langle 1,0\rangle} \frac{\partial F^{\langle 0,1\rangle}}{\partial t}\right.} \\
& \left.+\frac{1}{R^{2}} \frac{\partial \psi^{\langle 0,1\rangle}}{\partial \theta} \frac{\partial F^{\langle 1,0\rangle}}{\partial \theta}\right]_{r=R}, \\
p^{[1,1]}= & {\left[p^{\langle 1,1\rangle}+F^{\langle 1,0\rangle} \frac{\partial p^{\langle 1,0\rangle}}{\partial r}\right]_{r=R} } \\
= & \frac{\gamma}{R^{2}} \sum(n-1)(n+2) a_{n}^{\langle 1,0\rangle} P_{n}(\cos \theta) .
\end{aligned}
$$

By substituting $\psi^{\langle 0,1\rangle}, F^{\langle 1,0\rangle}$, and $F^{\langle 0,1\rangle}$ into the kinematic and normal boundary conditions, we obtain the ordinary differential equation for $a_{m}^{\langle(1,1)\rangle}(t)$ :

$$
\begin{aligned}
\ddot{a}_{m}^{\langle 1,1\rangle}(t)+\frac{m(m+1)(m-1)(m+2)}{n(n+1)(n-1)(n+2)} a_{m}^{\langle 1,1\rangle}(t) \\
=\frac{(2 m+1)}{2}\left[\frac{\omega_{m}^{\langle 1,0\rangle}}{\omega_{n}^{\langle 0,0\rangle}}\left(1+\frac{m}{n}\right)\left\langle P_{n}, P_{m}\right\rangle \cos t\right. \\
+\operatorname{sgn}(W) \frac{(2 n+1) P_{n}(0)}{(n-1)(n+2)}\left\langle(m-n+1) P_{n}^{2}\right. \\
\left.\left.+\frac{1}{n}\left(\frac{\partial P_{n}}{\partial \theta}\right)^{2}, P_{m}\right\rangle \cos t\right] .
\end{aligned}
$$

Given that $\left\langle P_{n}, P_{m}\right\rangle=0$ for $n \neq m, \omega_{m}^{\langle 1,0\rangle}$ is determined only when $n=m$.

If $n=m$, then the secular term should vanish:

$$
\begin{aligned}
& 2 \frac{\omega_{n}^{\langle 1,0\rangle}}{\omega_{n}^{\langle 0,0\rangle}}\left\langle P_{n}, P_{n}\right\rangle+\operatorname{sgn}(W) \frac{(2 n+1) P_{n}(0)}{(n-1)(n+2)} \\
& \quad \times\left\langle P_{n}^{2}+\frac{1}{n}\left(\frac{\partial P_{n}}{\partial \theta}\right)^{2}, P_{n}\right\rangle=0 .
\end{aligned}
$$

Thus, the correction to the order $\varepsilon_{1}$ is given by $\frac{\omega_{n}^{\langle 1,0\rangle}}{\omega_{n}^{\langle 0,0\rangle}}=-\operatorname{sgn}(W) \frac{(2 n+1)^{2} P_{n}(0)}{4(n-1)(n+2)}\left\langle P_{n}^{2}+\frac{1}{n}\left(\frac{\partial P_{n}}{\partial \theta}\right)^{2}, P_{n}\right\rangle$.
[1] L. Rayleigh, Proc. R. Soc. London 29, 71 (1879).

[2] H. Lamb, Hydrodynamics (Cambridge University Press, Cambridge, 1932).

[3] S. Chandrasekhar, Proc. London Math. Soc. s3-9, 141 (1959).

[4] H. H. K. Tang and C. Y. Wong, J. Phys. A: Math. Nucl. Gen. 7, 1038 (1974).

[5] A. Prosperetti, Q. Appl. Math. 34, 339 (1977).

[6] M. Strani and F. Sabetta, J. Fluid Mech. 141, 233 (1984).

[7] M. Strani and F. Sabetta, J. Fluid Mech. 189, 397 (1988).

[8] J. B. Bostwick and P. H. Steen, Phys. Fluids 21, 032108 (2009).

[9] J. B. Bostwick and P. H. Steen, J. Fluid Mech. 714, 312 (2013).
[10] J. B. Bostwick and P. H. Steen, J. Fluid Mech. 714, 336 (2013).

[11] C.-T. Chang, J. B. Bostwick, P. H. Steen, and S. Daniel, Phys. Rev. E 88, 023015 (2013).

[12] O. V. Voinov, Fluid Dynam. 11, 714 (1976).

[13] R. G. Cox, J. Fluid Mech. 168, 169 (1986).

[14] L. M. Hocking, J. Fluid Mech. 179, 267 (1987).

[15] I. S. Fayzrakhmanova and A. V. Straube, Phys. Fluids 21, 072104 (1987).

[16] D. V. Lyubimov, T. P. Lyubimova, and S. V. Shklyaev, Phys. Fluids 18, 012101 (2006). 
[17] D. V. Lyubimov, T. P. Lyubimova, and S. V. Shklyaev, Fluid Dynam. 39, 851 (2013).

[18] X. Noblin, A. Buguin, and F. Brochard-Wyart, Eur. Phys. J. E 14, 395 (2004).

[19] X. Noblin, A. Buguin, and F. Brochard-Wyart, Eur. Phys. J. Special Top. 166, 7 (2009).

[20] J. S. Sharp, D. J. Farmer, and J. Kelly, Langmuir 27, 9367 (2011).

[21] J. S. Sharp, Soft Matter 8, 399 (2012).

[22] F. Mugele and J.-C. Baret, J. Phys.: Condens. Matter 17, R705 (2005).

[23] T. D. Blake, A. Clarke, and E. H. Stattersfield, Langmuir 16, 2928 (2000).

[24] C. Quilliet and B. Berge, Curr. Opin. Colloid Interface Sci. 16, 34 (2001).

[25] F. Li and F. Mugele, Appl. Phys. Lett. 92, 244108 (2008).

[26] J.-Y. Yoon and R. L. Garrell, Anal. Chem. 75, 5097 (2003).

[27] H. B. Eral, D. M. Augustine, M. H. G. Duits, and F. Mugele, Soft Matter 7, 4954 (2011).

[28] P. Paik, V. K. Pamula, and R. B. Fair, Lab Chip 3, 253 (2003).

[29] F. Mugele, J.-C. Baret, and D. Steinhauser, Appl. Phys. Lett. 88, 204106 (2006)

[30] C.-P. Lee, H.-C. Chen, and M.-F. Lai, Biomicrofluidics 6, 012814 (2012).

[31] D. J. C. M. t Mannetje, C. U. Murade, D. van den Ende, and F. Mugele, Appl. Phys. Lett. 98, 014102 (2011).
[32] J. Hong, S. J. Lee, B. C. Koo, Y. K. Suh, and K. H. Kang, Langmuir 28, 6307 (2012).

[33] J. M. Oh, S. H. Ko, and K. H. Kang, Langmuir 24, 8379 (2008).

[34] M.-F. Lai, C.-P. Lee, C.-N. Liao, and Z.-H. Wei, Appl. Phys. Lett. 94, 154102 (2009).

[35] S. Dash, N. Kumari, and S. V. Garimella, J. Micromech. Microeng. 22, 075004 (2012).

[36] J. Hong, Y. K. Kim, K. H. Kang, J. Kim, and S. J. Lee, Sens. Actuator B-Chem. 190, 48 (2014).

[37] J. M. Oh, S. H. Ko, and K. H. Kang, Phys. Fluids 22, 032002 (2010).

[38] M. S. Plesset, J. Appl. Phys. 25, 96 (1954).

[39] J. A. Tsamopoulos and R. A. Brown, J. Fluid Mech. 127, 519 (1983).

[40] Q. Feng and K. V. Beard, Proc. R. Soc. London A 430, 133 (1990).

[41] J. Buehrle, S. Herminghaus, and F. Mugele, Phys. Rev. Lett. 91, 086101 (2003).

[42] H. G. Weller, G. Tabor, H. Jasak, and C. Fureby, Comput. Phys. 12, 620 (1998).

[43] S. Afkhami, S. Zaleski, and M. Bussman, J. Comput. Phys. 228, 5370 (2009).

[44] J. K. Park and K. H. Kang, Phys. Fluids 24, 042105 (2012).

[45] E. Olsson and G. Kreiss, J. Comput. Phys. 210, 225 (2005).

[46] E. Olsson, G. Kreiss, and S. Zahedi, J. Comput. Phys. 225, 785 (2007).

[47] S. Mettu and M. K. Chaudhury, Langmuir 28, 14100 (2012). 